\section{E-073 IMPACT OF DIABETES WITH COMPLICATIONS ON SHORT-TERM IN-HOSPITAL OUTCOMES IN PATIENTS UNDERGOING SPINAL FUSION PROCEDURES}

T Colburn*, D Schirmer, Z Rose-Reneau, B Wright. Kansas City University of Medicine and Biosciences, Kansas City, MO

\subsection{6/neurintsurg-2019-SNIS.148}

Background Spinal fusion is a surgical procedure utilized to treat or alleviate several spinal diagnoses including: tumor, spinal stenosis, degenerative disc disease, scoliosis, and spondylolisthesis. Previous studies have explored clinical outcomes for this intervention. This study aims to further investigate shortterm outcomes of patients after spinal fusion by looking at the impact of diabetes with complications.

Methods This retrospective cohort study utilized data from the Nationwide Inpatient Sample (NIS) to identify adult patients $(18+)$ from 2012-2015 who underwent spinal fusion. ICD-9 codes identified these patients; specifically patients diagnosed with diabetes and associated complications. Any patients missing important clinical identifiers (age, gender, cause of death) and patients without spinal fusion intervention were excluded. Data analyses assessed hospital length of stay (LOS), inpatient charges, average age of admission and mortality rate.

Results Of the 290,752 patients that underwent spinal fusion procedure, 1,092 had the diagnosis of diabetes with complications (DMCx).

Mean mortality rate of patients was significantly increased (1.8\%, DMCx vs. 0.6\%, without DMCx, p = 0.0001)

LOS in patients with DMCx who underwent spinal fusion was significantly increased (7.44 days, DMCx vs. 4.01 days, no DMCx p $=0.0001$ )
Total hospital charges were significantly increased $(\$ 135,783.00, \mathrm{DMCx}$ vs. $\$ 98,231.18$, no $\mathrm{DMCx}, \mathrm{p}=$ $0.0001)$

Mean age of patients at admission was increased (63.6, DMCx vs. 57.67, no DMCx, p =0.0001)

Conclusion Patients with complicated diabetes who undergo a spinal fusion procedure suffer from increased mortality rate, LOS, total hospital charges, and age at admission. This study aims to provide physicians with information in the management of patients with complicated diabetes that undergo spinal fusion procedures. Peri-procedural patient optimization could provide a potential avenue to lower LOS, total in-hospital charges, and mortality in patients.

Disclosures T. Colburn: None. D. Schirmer: None. Z. RoseReneau: None. B. Wright: None.

\section{E-074 TRANSRADIAL APPROACH FOR THE TREATMENT OF A SACRAL DURAL ARTERIOVENOUS FISTULA: HOW FAR CAN WE GO?}

E Orru'*, C Tsang, J Klostranec, V Mendes Pereira. Neuroradiology, Toronto Western Hospital, Toronto, ON, Canada

\subsection{6/neurintsurg-2019-SNIS.149}

Sacral dural arteriovenous fistulae (SDAVFs) are rare, constituting no more than $10 \%$ of all spinal dural fistulae. They are most commonly fed by the lateral sacral artery (LSA), a branch of the internal iliac artery (IIA). Catheterization of this vessel requires either a cross- over at the aortic bifurcation in
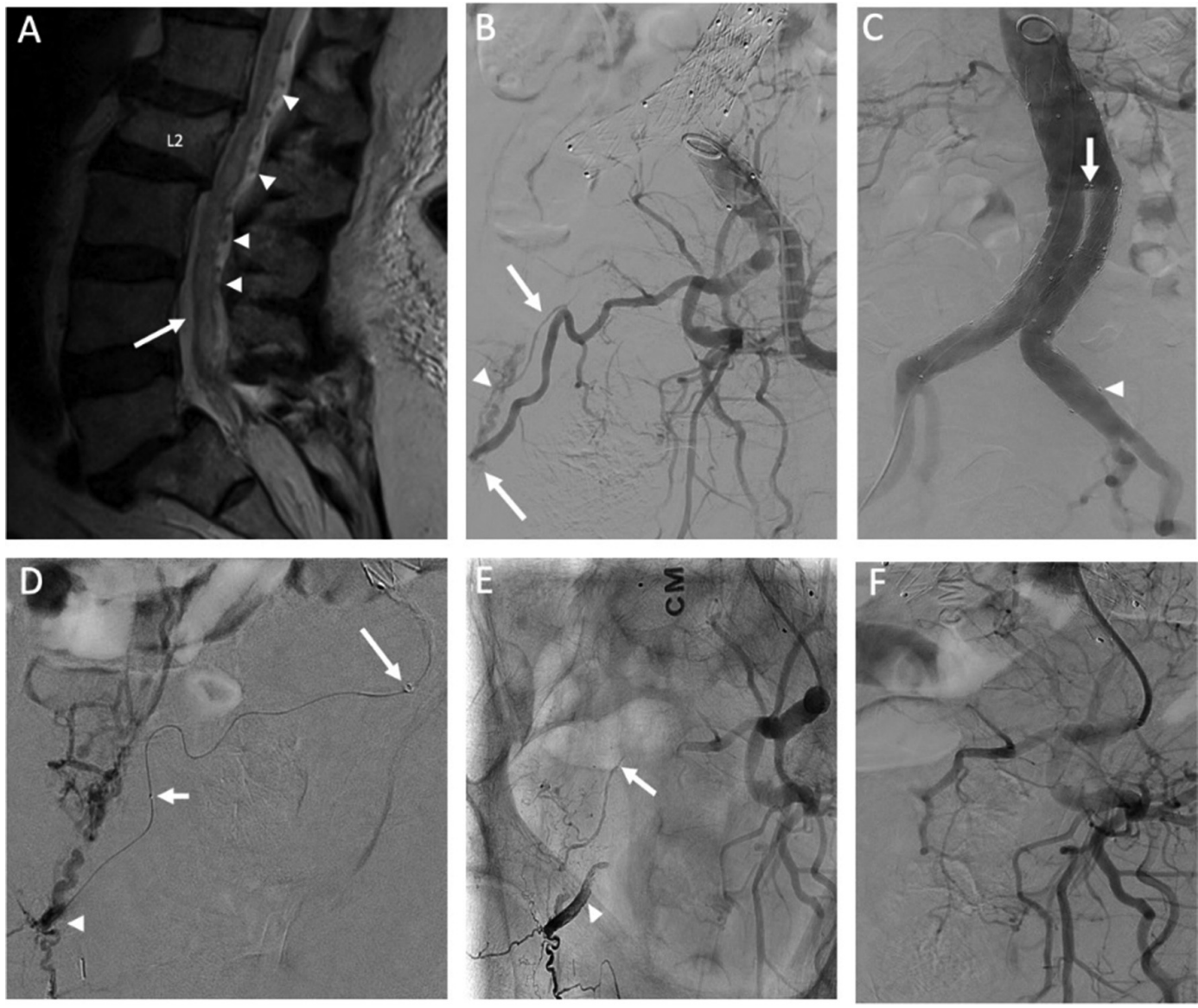

Abstract E-074 Figure 1 